\title{
SURTOS DE SALMONELOSE NOTIFICADOS NO PERÍODO DE JANEIRO DE 2009 A JULHO DE 2014 NO ESTADO DO PARANÁ, BRASIL
}

\author{
Débora de Almeida Gabaron \\ Luciana Kazue Otutumi ${ }^{2}$ \\ Ranulfo Piau Júnior ${ }^{2}$
}

GABARON, D. de A.; OTUTUMI, L. K.; PIAU JÚNIOR, R. Surtos de salmonelose notificados no período de janeiro de 2009 a julho de 2014 no estado do Paraná, Brasil. Arq. Ciênc. Vet. Zool. UNIPAR, Umuarama, v. 18, n. 1, p. 33-37, jan./ mar. 2015.

RESUMO: A incidência de doenças relacionadas ao consumo de alimentos vem aumentando a cada dia, o que demonstra a importância de estudos que avaliem os agentes etiológicos envolvidos para que se possa traçar estratégias de controle do aparecimento de surtos. A salmonelose é uma enfermidade de grande importância para a saúde pública, sendo importante a sua investigação, visto que seu agente etiológico, a Salmonella spp. é um dos principais micro-organismos envolvidos em surtos de toxinfecções alimentares. Sendo assim, o objetivo do trabalho foi avaliar o número de surtos alimentares ocasionados por Salmonella spp., notificados no Estado do Paraná entre janeiro de 2009 a julho de 2014. Neste período foram notificados 105 surtos de toxinfecção alimentar e desses 19 casos (18,09\%) foram confirmados em laboratório como sendo Salmonella spp. No entanto, dentre os surtos de Salmonella spp. ocorridos no período não houve nenhuma notificação de morte ocasionada por este agente. Os resultados demonstram a necessidade de realização de treinamento em Boas Práticas de Fabricação (BPF) para os manipuladores, além do planejamento de ações em educação sanitária para a população consumidora, e a necessidade de um maior controle dos surtos alimentares, para que se possa contribuir efetivamente no controle desse tipo de doença por meio da redução da população exposta ao risco.

PALAVRAS-CHAVE: Boas práticas de fabricação. Doenças transmitidas por alimentos. Segurança alimentar. Vigilância sanitária.

\section{SALMONELLOSIS OUTBREAKS REPORTED FROM JANUARY 2009 TO JULY 2014 IN THE STATE OF PARANÁ, BRAZIL}

\begin{abstract}
The incidence of diseases related to food consumption is increasing, which shows the importance of studies assessing the etiologic agents involved so that strategies to control outbreaks can be developed. Salmonellosis is a disease of great importance to public health, and its investigation is important, since its etiological agent - Salmonella spp. - is one of the main microorganisms involved in food poisoning outbreaks. Thus, the objective of this study was to evaluate the number of food outbreaks caused by Salmonella spp., reported in the state of Paraná from January 2009 to July 2014. During this period, a total of 105 food poisoning outbreaks were reported, and from these, 19 cases $(18.09 \%)$ were confirmed in laboratory as Salmonella spp. However, among the outbreaks of Salmonella spp. occurring during the period, there was no notification of death caused by this agent. The results demonstrate the need to have training in Good Manufacturing Practices (GMP) for handlers, in addition to planning activities in health education for the consuming population, as well as the need for greater control of food outbreaks, for effectively contributing to control this disease by reducing the population exposed to the risk. KEY WORDS: Good manufacturing practices. Foodborne illness. Food safety. Health surveillance.
\end{abstract}

\section{BROTES DE SALMONELOSIS NOTIFICADOS EN EL PERIODO DE ENERO DE 2009 A JULIO DE 2014 EN EL ESTADO DE PARANÁ, BRASIL}

RESUMEN: La incidencia de enfermedades relacionadas con el consumo de alimentos está aumentando a cada día, lo que demuestra la importancia de estudios que evalúen los agentes etiológicos implicados, para que se pueda trazar estrategias de control del aparecimiento de brotes. La salmonelosis es una enfermedad de gran importancia para la salud pública, es importante su investigación, puesto que su agente etiológico, la Salmonella spp. es uno de los principales microorganismos implicados en brotes de intoxicaciones alimentares. Por lo tanto, el objetivo de este estudio ha sido evaluar el número de brotes por alimentos causados por Salmonella spp., reportados en el Estado de Paraná entre enero de 2009 a julio de 2014. Durante este período se reportaron 105 brotes de intoxicación alimentar, y de esos, 19 casos (18,09\%) fueron confirmados en laboratorio como Salmonella spp. Sin embargo, entre los brotes de Salmonella spp. producidos durante el período no hubo ninguna notificación de muerte causada por este agente. Los resultados demuestran la necesidad de llevar a cabo la capacitación en Buenas Prácticas de Manufactura (BPF) para los manipuladores, además de planificaciones de acciones en educación

DOI: https://doi.org/10.25110/arqvet.v18i1.2015.5367

${ }^{1}$ Tecnóloga em Alimentos, Mestranda em Ciência Animal da Universidade Paranaense - UNIPAR, e-mail: deboragabaron@hotmail.com. Praça: Curitiba, n ${ }^{\circ}$ 16. CEP: 87450-000, Tuneiras do Oeste, Paraná;

${ }^{2}$ Professor do curso de Medicina Veterinária e do Mestrado em Ciência Animal da Universidade Paranaense - UNIPAR, Umuarama, Paraná. Praça Mascarenhas de Moraes, $n^{\circ}$ 4282. Campus Sede. CEP: 87502-210, Umuarama, Paraná. 
sanitaria para la población consumidora, y la necesidad de un mayor control de los brotes alimentares, para que se pueda contribuir efectivamente en el control de ese tipo de enfermedad, por medio de la reducción de la población expuesta al riesgo. PALABRAS CLAVE: Buenas prácticas de fabricación. Enfermedades transmitidas por alimentos. Seguridad alimentar.

Vigilancia sanitaria.

\section{Introdução}

As Doenças Transmitidas por Alimentos (DTA's) são atribuídas à ingestão de alimentos ou água contaminadas por bactérias, vírus, parasitos, toxinas, príons, agrotóxicos, produtos químicos e metais pesados (HOBBS; ROBERTS, 1999). Essas DTA's podem se manifestar na forma de infecções, intoxicações e toxinfecções alimentares. Especificamente em relação às toxinfecções alimentares, Wingert (2012) enfatiza que as mesmas resultam da ingestão de alimentos com certa quantidade de micro-organismos causadores de doenças, capazes de produzir ou liberar toxinas após serem ingeridos.

A investigação de um surto de DTA se embasa em três eixos principais: (1) a investigação epidemiológica propriamente dita, por meio de formulários com entrevistas aos envolvidos no surto, para identificar o veículo de transmissão e o provável agente etiológico; (2) a investigação laboratorial, com a coleta de amostras clínicas de pacientes, alimentos e água, para confirmação e (3) a investigação ambiental, ou seja, averiguação do local de ocorrência do surto para se detectar os fatores contribuintes que possibilitaram o surgimento do mesmo (SANTA CATARINA, 2006).

Segundo Perdoncini et al. (2014), a Salmonella spp. é uma bactéria altamente envolvida em DTA's no estado do Rio Grande do Sul, o que fez com que se aumentasse o número de pesquisas com o objetivo de se diminuir a transmissão dessa bactéria aos seres humanos. No estado do Paraná, Almeida et al. (2013) relataram que a Salmonella spp. esteve envolvida em aproximadamente $10 \%$ dos surtos alimentares.

Mais especificamente, Kottwitz et al. (2013) observaram que dentre os 254 surtos de salmonelose ocorridos entre 2005 a 2008 no estado do Paraná, 207 (81,49\%) casos tiveram o diagnóstico comprovado mediante confirmação laboratorial.

As bactérias do gênero Salmonella, são bacilos gram negativos, pertencentes à família Enterobacteriaceae, não formadores de esporos, anaeróbios facultativos. Em sua maioria são movimentadas por flagelos, à exceção da $S$. Pullorum e da $S$. Gallinarum, que são imóveis. A temperatura ótima de crescimento é em média de $38^{\circ} \mathrm{C}$, sendo destruídas a temperaturas acima dos $60^{\circ} \mathrm{C}$, e não apresentando crescimento sob temperaturas abaixo de $5^{\circ} \mathrm{C}$ (FORTUNA; FRANCO, 2005, BRASIL, 2009).

Todas as espécies paratíficas de Salmonella são patógenas ao homem (JAY, 2005), tornando-se uma enfermidade de extrema importância mundial (PICKLER, 2012), caracterizando-se como uma infecção aguda (SIMÕES et al., 2010), sendo conhecidos mais de 2.500 sorotipos de Salmonella, com apenas 80 a 90 os mais comuns em casos de infecções nas aves e nos humanos (BENEZ, 2001; PELCZAR et al., 1996).

Segundo Silva (2010), a principal forma de transmissão da Salmonella spp. é pela via fecal-oral, por meio da ingestão de água ou alimentos contaminados ou por contato direto com animais infectados. Segundo o mesmo autor, outras vias de transmissão também são relatadas como, por exemplo, o contato com superfícies contaminadas com matéria orgânica, água ou fezes contendo o micro-organismo, o qual pode sobreviver por longos períodos.

Levando-se em consideração que a Salmonella spp. continua envolvida em surtos de DTA's, há a necessidade de um rastreamento maior a nível nacional em relação à presença dessa bactéria em alimentos prontos para o consumo que subsidiem os órgãos de saúde pública responsáveis definirem critérios e padrões microbiológicos para o agente (FAI et al., 2011).

Embora a maioria dos casos de pessoas doentes se recupere sem tratamento, em algumas, a diarreia pode ser tão agressiva que a hospitalização é necessária, podendo acontecer da infecção se disseminar dos intestinos para a corrente sanguínea e daí para outras partes do corpo, e como consequência ocasionar a morte. No entanto, as pessoas idosas, crianças e as pessoas com o sistema imune-deprimidas são as mais vulneráveis a desenvolver a forma mais severa da doença (SIMÕES, 2010).

Em relação aos alimentos, os mais suscetíveis à contaminação por salmonelas são as carnes frescas, principalmente carcaças de aves, além de leite, queijo e ovos (PINTO, 1996).

Dessa forma, o objetivo deste trabalho foi fazer um estudo retrospectivo dos surtos alimentares ocasionados por Salmonella spp., notificados no Estado do Paraná entre janeiro de 2009 a julho de 2014, na região de abrangência das 22 regionais de saúde do Paraná, Brasil.

\section{Material e Métodos}

\section{Local de realização e amostragem}

Foi realizado um levantamento de dados oriundos das 22 Regionais de Saúde, da Secretaria Estadual de Saúde do Estado do Paraná, Brasil (Figura 1), englobando os casos de surtos alimentares registrados dentro do período compreendido entre os anos de 2009 a 2014, a partir dos informes semanais epidemiológicos registrados no período de janeiro de 2009 a julho de 2014 .

O levantamento foi feito com base nos dados da frequência de salmonelose, especificados por análise de coprocultura e separando-os por faixa etária.

Segundo dados do IPARDES (2014), o Paraná é uma região territorial grande, que conta com 399 municípios e faz divisa com os estados de São Paulo, Santa Catarina e Mato Grosso do Sul, fronteira com a Argentina e o Paraguai e limite com o Oceano Atlântico, ocupando uma área de $1999.880 \mathrm{~km}^{2}$. 
Figura 1: Mapa do estado do Paraná, Brasil, demonstrando a localização das 22 Regionais de Saúde do Estado.

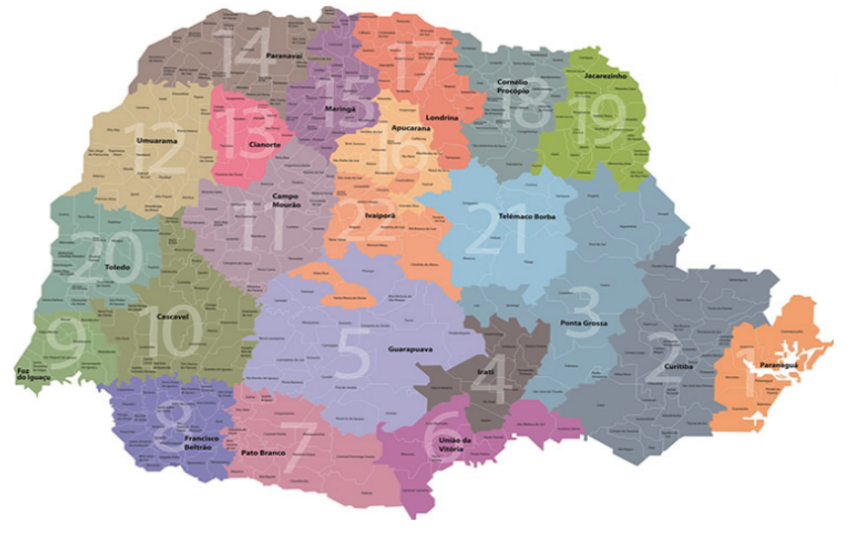

Fonte: PARANÁ, 2014.

\section{Resultados}

Dos surtos ocorridos entre janeiro de 2009 a julho de 2014, as notificações foram seguidas de confirmação laboratorial por meio do isolamento de Salmonella spp. para um diagnóstico específico. Dos 105 surtos de DTA’s ocorridos, não houve nenhuma notificação de morte ocasionada por esse agente patogênico no período de estudo. No entanto, dentre os 105 surtos de DTA's ocorridos no período, 19 casos foram diagnosticados como sendo causados por contaminação por Salmonella spp.

Em relação à idade, no período de 2009 a 2012, não foi realizado análise correlacionando o número de casos com a faixa etária, devido à falta de dados nos Informes semanais. Dentre os casos notificados nos anos de 2013 e 2014, quatro ocorreram em pessoas com idade variando entre cinco a 14 anos, seis casos em pessoas entre 15 a 29 anos e nove casos em pessoas entre 30 a 40 anos de idade. A Figura 2 ilustra o percentual de surtos de salmonelose, em função da faixa etária.

Figura 2: Percentual de indivíduos envolvidos em surtos por Doenças Transmitidas por Alimentos, ocasionados por Salmonella spp., de acordo com a faixa etária, nas $22^{\mathrm{a}}$ Regionais de Saúde do Estado do Paraná, no período de 2009-2014.

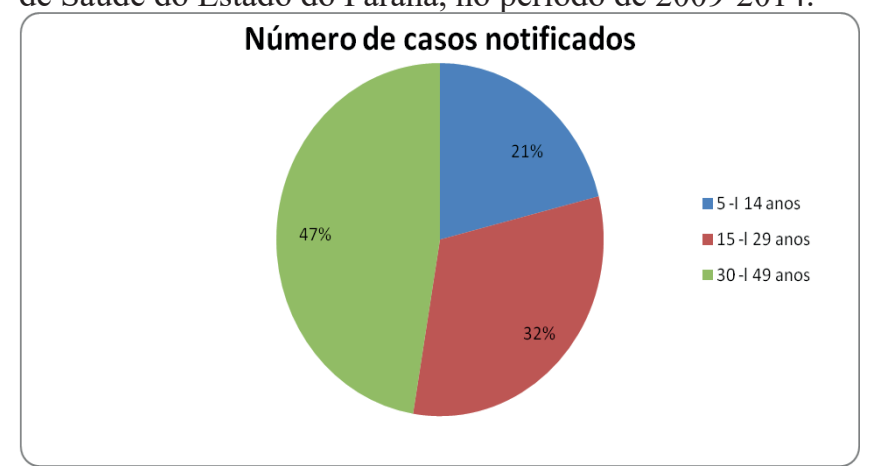

Fonte: Autores.

\section{Discussão}

No Brasil, diversos são os relatos de surtos de toxinfecções causadas por Salmonella spp. (KOTTWITZ et al., 2010) e a gastroenterite ocasionada por Salmonella representa um problema de saúde pública mundial (ALMEIDA et al.
2013).

De acordo com Almeida et al (2013), os indivíduos entre 20 e 49 anos de idade foram os mais frequentemente envolvidos em surtos de DTA's representando 49,50\% das ocorrências, em um estudo abrangendo dados da segunda Regional de Saúde do Paraná, Brasil, o que está próximo aos resultados encontrados no presente trabalho.

Resultados semelhantes foram encontrados por Madalosso et al. (2008) os quais observaram que a faixa etária mais acometida entre os doentes foi de 20 a 49 anos (87,5\%), em surtos alimentares causados por Salmonella spp. em São Paulo, SP.

Por outro lado, Peresi (1998) demonstrou em seu estudo que os elevados índices de internações eram compostos por crianças e idosos, correspondendo a $66,7 \%$ dos casos, o que representa elevados custos econômicos e sociais. Neste contexto, ressalta-se a gravidade da infecção em faixas etárias extremas, enfatizando os cuidados com a alimentação.

Segundo Santos e Gonçalves (2010), a elevada taxa de surtos causados por micro-organismos patogênicos é um dos indicadores de falta de higiene dos manipuladores e na manipulação dos alimentos, aliada a variáveis como a falta ou a inadequada refrigeração, contaminação cruzada e armazenamento inadequado dos alimentos.

Os alimentos, principalmente os de origem animal estão intimamente relacionados aos casos de surtos de salmonelose humana, onde representam excelentes meios para o desenvolvimento bacteriano, devido à grande variedade de nutrientes, à elevada atividade de água, à baixa acidez $(\mathrm{pH}$ entre 5,5 e 7,0) e, em muitos casos, serem armazenados sem refrigeração adequada (FORSYTHE, 2002; HOBBS; ROBERTS, 1999), no entanto, no presente trabalho, não foram identificados os alimentos envolvidos nos surtos de salmonelose durante o período de estudo.

Cabe salientar que o número de surtos notificados de doenças transmitidas por alimentos (DTA's) representa apenas a ponta de um iceberg, se comparado com o total de ocorrências (FORSYTHE, 2002), visto que existe uma sonegação de dados durante o processo de notificação, o que justifica o número significativamente reduzido em nosso estudo.

Germano et al. (1993), enfatizam ainda que apenas $10 \%$ do número real de surtos de toxinfecções alimentares são confirmados, em decorrência do atual estado de desenvolvimento dos serviços de vigilância epidemiológica, além da falta de conscientização da população frente ao grave problema existente.

A falta de dados disponíveis no sistema de acesso ao público dificulta a análise completa desse levantamento, já que somente dados de quantidade e idade estão disponíveis nos Informes, porém outros dados importantes como fator causal, sexo e zona de ocorrência não são relatados, dificultando assim o levantamento mais detalhado dos dados, e não permitindo a prevenção adequada dessa doença.

Por outro lado, ressalta-se que a alimentação de acordo com os padrões higiênicos satisfatórios e adoção de boas práticas de fabricação é condição fundamental para a promoção e manutenção da saúde (NADVORNY et al., 2004; SOARES et al., 2006).

Vale ressaltar o papel da Vigilância Sanitária não apenas na fiscalização, mas também na sua atuação como multiplicador das boas práticas higiênico-sanitárias mediante 
atividades educativas permanentes dirigidas aos consumidores, alertando para os riscos representados pela manipulação inadequada (VALENTE, 2004).

As boas práticas de fabricação formam um dos componentes fundamentais para a segurança alimentar, pois onde se tem o risco de perigos, estes devem ser controlados, garantindo a sua integridade e eficácia e, adquirindo assim a segurança alimentar.

Além disso, uma redução expressiva do número de surtos representaria uma diminuição significativa de gastos com medicamentos, internações, além de perdas de dias de serviço, entre outras perdas não só de níveis econômicos como também sociais.

\section{Conclusão}

Com base nos 19 casos de salmonelose notificados no período de janeiro de 2009 a julho de 2014, demonstra-se a necessidade de realização de treinamento em Boas Práticas de Fabricação para os manipuladores, ministrados pelos profissionais da saúde (Vigilância Sanitária dos Alimentos), além do planejamento de ações em educação sanitária para a população consumidora e a necessidade de um maior controle dos surtos alimentares, para que se possa contribuir efetivamente no controle dos mesmos e reduza a população exposta ao risco.

Além disso, é necessário uma fiscalização mais rigorosa e implementação de programas de controle de qualidade que garantam a oferta de alimentos prontos para o consumo, seguros à saúde do consumidor. A elaboração de cartilhas, folhetos e vídeos educativos podem ser de extrema valia para a conquista de resultados satisfatórios visando a vigilância e a promoção de saúde.

\section{Referências}

ALMEIDA, J. C. et al. Perfil epidemiológico de casos de surtos de doenças transmitidas por alimentos ocorridos no Paraná, Brasil. Semina: Ciências Biológicas e da Saúde, Londrina, v. 34, n. 1, p. 97-106, jan./jul. 2013.

BRASIL. Manual de legislação: Programas nacionais de saúde animal do Brasil. Brasília: Ministério da Agricultura, Pecuária e Abastecimento, 2009. p. 441.

BENEZ, S. M. Aves: criação, clínica, teoria, prática silvestres, ornamentais, avinhados. Robe: São Paulo, 2001. p. 413-416.

CORRÊA, W. M.; CORRÊA, C. N. M. Enfermidades infecciosas dos mamíferos domésticos. Rio de Janeiro: Médica e Científica, n. 2, p. 163-174, 1992.

FAI, A. E. C. et al. Salmonella spp e Listeria monocytogenes em presunto suíno comercializado em supermercados de Fortaleza (CE, Brasil): fator de risco para a saúde pública. Ciência \& Saúde Coletiva, v. 16, n. 2, p. 657-662, 2011.

FORSYTHE, S. J. Microbiologia da segurança alimentar. 2. ed. Porto Alegre: Artmer, 2002. 424 p.
FORTUNA, J. L.; FRANCO, R. B. Pequeno dossiê epidemiológico da Salmonella, como causadora de infecções alimentares. Higiene Alimentar, v. 128, p. 33-43, 2005.

GERMANO, P. M. L. et al. Prevenção e controle das toxinfecções de origem alimentar. Higiene Alimentar, v. 7, n. 27 , p. 6-11, 1993.

HOBBS, B. C.; ROBERTS, D. Toxinfecções e controle higiênico-sanitário de alimentos. São Paulo: Varela, 1999. p. 376 .

IPARDES (Instituto Paranaense de Desenvolvimento Econômico e Social). Paraná em números. Disponível em: $<$ http://www.ipardes.gov.br/index.php?pg conteudo $=1 \&$ cod_conteudo $=1>$. Acesso em: 27 set. 2014 .

JAY, J. M. Microbiologia de alimentos. 6. ed. Porto Alegre: Artmed, 2005.

KOTTWITZ, L. B. M. et al. Avaliação epidemiológica de surtos de salmonelose ocorridos no período de 1999 a 2008 no Estado do Paraná, Brasil. Acta Scientiarum, v. 32, n. 1, p. 9-15, 2010.

MADALOSSO, G. et al. Surto alimentar por Salmonella enterica sorotipo Enteritidis em restaurante, São Paulo, SP dezembro de 2006. Boletim Epidemiológico Paulista, v. 5 , n. 55, 2008. Disponível em: <http://www.cve.saude.sp.gov. br/agencia/bepa55_salmonella.htm>. Acesso em: 14 jan. 2015 .

MARTINS, R. F. B. Avaliação da prevalência da implementação do sistema HACCP e dos seus efeitos nos indicadores de segurança alimentar, em instituições de acolhimento a crianças e idosos, na região do Porto. 2013. 327 f. Tese (Doutorado em Saúde Pública) Universidade de Santiago de Compostela, Galiza - Espanha, 2013.

NADVORNY, A.; FIGUEIREDO, D. M. S.; SCHMIDT, V. Ocorrência de Salmonella sp. em surtos de doenças transmitidas por alimentos no Rio Grande do Sul em 2000. Acta ScientiaeVeterinariae, v. 32, n. 1, p. 47-51, 2004.

PARANÁ. Secretaria de Saúde. Regionais de Saúde. Disponível em: <http:/www.saude.pr.gov.br/modules/ conteudo/conteudo.php? conteudo $=2752>$. Acesso em: 12 out. 2014.

PELCZAR, M.; REID, R.; CHAN, E. C. S. Microbiologia: conceitos e aplicações. São Paulo: MAKRON, 1996.

PERDONCINI, G. et al. Salmonella spp. em ovos produzidos em sistema agroecológico. Revista Agrocientífica, n. 1, v. 1, p. 33-42, 2014.

PERESI, J. T. M. et al. Surtos de enfermidades transmitidas por alimentos causados por Salmonella enteritidis. Revista de saúde Pública, v. 32, p. 477-483, 1998. 
PICKLER, L. et al. Avaliação microbiológica, histológica e imunológica de frangos de corte desafiados com Salmonella enteritidis e Minnesota e tratados com ácidos orgânicos.

Pesquisa Veterinária Brasileira, v. 32, n. 1, p. 27-36, 2012.

PINTO, A. F. M. A. Doenças de origem microbiana transmitidas pelos alimentos. Millenium, n. 4, p. 91-100, 1996.

ROCHA e SILVA, R. C. et al. O pombo (Columba livia) como agente carreador de Salmonella spp. e as implicações em saúde pública. Arquivo Instituto Biológico, v. 81, n. 2, p. 189-194, 2014.

ROITMAM, I.; TRAVASSOS, L.; AZEVEDO, J. L. Tratado de microbiologia. São Paulo: Manole, 1987. p. 181.

SANTA CATARINA Secretaria de Estado da Saúde de Santa Catarina. Manual de orientação para investigação em surtos de DTA. Florianópolis, 2006.

SANTA CATARINA. Hospital Universitário/UFSC. Serviço de análises clínicas: laboratório de microbiologia. Manual de coleta para exames microbiológicos. Florianópolis, 2014

SANTOS, I. C.; GONÇALVES, E. C. B. A. Qualidade de carnes in natura na recepção de uma rede de supermercados e implantação de ações educativas para os manipuladores dos produtos. Revista Higiene Alimentar, v. 24, n. 183, p. 38-44, 2010.

SANTOS, L. R. et al. Salmonella enteritidis isoladas de amostras clínicas de humanos e de alimentos envolvidos em episódios de toxinfecções alimentares, ocorridas entre 1995 e 1996 no estado do Rio Grande do Sul. Higiene Alimentar, v. 16, p. 93-99, ano.

SILVA, M. A. et al. A importância da ordem ciconiiformes na cadeia epidemiológica de Salmonella spp. para a saúde pública e a conservação da diversidade biológica. Pesquisa Veterinária Brasileira, v. 7, n. 30, p. 573-580, 2010.

SIMÕES, M. et al. Salmonella enteriditis: importância do inquérito epidemiológico, análise de alimentos e coprocultura na elucidação de 167 surtos alimentares. Revista Instituto Adolfo Lutz, v. 4, n. 69, p. 497-502, 2010.

SOARES, A. G. et al. Boas práticas de manipulação em bancos de alimentos. Rio de Janeiro, Embrapa Agroindústria de Alimentos, 2006.

VALENTE, D. P. Avaliação higiênico-sanitária e físicoestrutural dos supermercados de uma cidade do Sudeste do Brasil. Revista Brasileira de Epidemiologia, v. 7, n. 1, p. 80-87, 2004.

WINGERT, C. Avaliação das condições higiênico- sanitárias dos serviços de alimentação de um shopping Center do município de Porto Alegre. 2012. 54 f. Dissertação (Mestrado em Ciências Veterinárias, especialidades na área de Bacteriologia Aplicada) Universidade Federal do Rio Grande do Sul, Porto Alegre, 2012. 